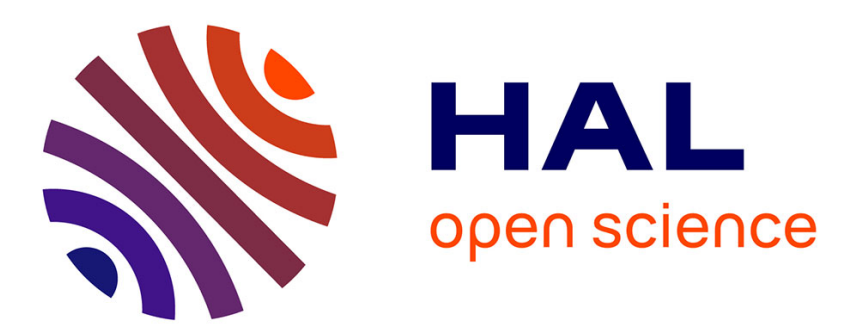

\title{
Data Window Aggregation techniques for Energy Saving in Wireless Sensor Networks
}

\author{
Somasekhar Kandukuri, Jean-Mickaël Lebreton, Denis Genon-Catalot, Nour
}

Murad, Richard Lorion

\section{- To cite this version:}

Somasekhar Kandukuri, Jean-Mickaël Lebreton, Denis Genon-Catalot, Nour Murad, Richard Lorion. Data Window Aggregation techniques for Energy Saving in Wireless Sensor Networks. ISCC 2016

- The twenty first IEEE Symposium on Computers and Communications, IEEE Computer Society, IEEE Communications Society, MDS LAb Messina, Jun 2016, Messina, Italy. hal-01344997

\section{HAL Id: hal-01344997 \\ https://hal.science/hal-01344997}

Submitted on 5 Apr 2018

HAL is a multi-disciplinary open access archive for the deposit and dissemination of scientific research documents, whether they are published or not. The documents may come from teaching and research institutions in France or abroad, or from public or private research centers.
L'archive ouverte pluridisciplinaire HAL, est destinée au dépôt et à la diffusion de documents scientifiques de niveau recherche, publiés ou non, émanant des établissements d'enseignement et de recherche français ou étrangers, des laboratoires publics ou privés. 


\section{Data Window Aggregation Techniques for Energy Saving in Wireless Sensor Networks}

\author{
Somasekhar Kandukuri, Jean Lebreton, \\ Nour Murad, Richard Lorion \\ Laboratory of Encrgy, Electronics and Process $\left(L E^{2} P\right)$ \\ University de La Reunion, 15 avenue Rene Cassin CS 92003 \\ 97744 Saint Denis CEDEX 9, Reunion * France \\ Enail: somaxckhar.kandukuri@univ-reunion. fr
}

\author{
Denis Genon-Catalot \\ University of Grenoble Alpes, \\ System Design and Integration Laboratory (LCS) \\ $F_{*} 26902$, Grenoble, France \\ Email: denis.genon $x$ catalot@iut $*$ valence, $\mathrm{fr}$
}

\begin{abstract}
Abstruct-Redundant data transmissions are likely to happen repeatedly in Wireless Sensor Networks (WSNs). According to the literature survey, energy-effictency predominately relies on data aggregation rather than routing or duty-cycling approaches. As data redundancy dominates the power usage in communication costs, in order to dentify and reduce the redundant data transmissions by individual nodes we propose a hybrd data aggregative window function (DAWD) algorithn for exploting hoth spatial and temporal data redundanctes in WSN. Furthermore, the proposed novel approach aims to process the hybrid filtration using both compressive and prediction based techiques in sensor nodes (SN) as well as in cluster-head (CH) nodes. In this regard, the experimental study case of this work show that the DAWF mechanism can suppress a huge amount of temporal redundant dafa transmissions in sensor nodes while providing reliable data messages towards the lase station (BS). Moreover, DAWF CH can also suppress a large amount of spatial redundancies by utiling the optinum DAWF parameters of the $\mathrm{CH}$ node.
\end{abstract}

Keyords-Predictive modeling, Data ageregation, Sensor networks, Data window, Spatio-tenporal, Correlations, Energy saving.

\section{INTRODUCTION}

In Wireless Sensor Networks (WSNs), if sensor nodes are used to have similar application tasks to monitor a set of physical quantities compared to the individual tasks, then the nodes may generate a large amount of redundant samples in both time and space. This is also observed in the analysis of real datasets from $[1,2]$ in both indoor and outdoor deployments. In [3], the authors are constructed the data models and reported parameters instead of raw sample values.

The cnergyefficiency issue in WSNs can also be achieved by exploiting the both inherent temporal and spatial redundancics (SRs) in data, such as temperature, humidity light and pressure exhibit the slight variations slowly over the period of time [4]. Moreover, this prospect may also help to balawe the uxage of node computational capabilitics, which profitably extracts the spatio-temporal models for avoiding the unnecessary sensing. The distributed nature of datadriven approaches has been well-studied in the literature [5-9]. Since, it is necessary to use an effective data redundarcy or simple prediction methods, in order to avoid the redundant data values to ensure the reliability in periodic or delay-tolerant network applications $[5,6]$.
The main idea of this work consists in exploiting temporal redundancies (TRs) in every node and their spatial redurdan. cies or correlations in cluster head $(\mathrm{CH})$ nodes. This prospect emphasizes the spationtemporal redundancies of measured physical quantities in order to compute the given spatio. temporal models, which then consecutively reduces the overall amount of required transmissions. The follow up of our A-DAWF protocol in [10]. which is framed by exploiting the temporal redundancies in every node using simple and inexpensive window aggregative models based on Intel lab datasets. In order to explore the in depth research work for exploiting the spationtemporal correlations in various physteal quantities of the nodes. We then present the datanaggregatiwe window function (DAWF) mchanism by exploiting spatial redundancies of the nodes in $\mathrm{CHs}$, which we present as a preliminary study cases of the $\mathrm{CHs}$ model behaviors. Our proposition has been tested on real datasets obtained from tre of our collaborative lab as LCIS, DREAL project dati sets, and the data collected from both indoor and outdoor environments of the thermal renovations of housing estates. DAWF mechanism improwes the encrgy efficiency in sensor nodes by showing that the overall required sensor readings can be redwed as well as their redundant data transmissions. The organization of this paper is as follows, section 2 describes the literature survey of several energy efficient data collection and gathering protocols. System models and implementations of our work hawe been presented in section 3 . Section 4 validates the simulation results to demonstrate that the sensor nodes can generate a huge amount of temporal redurdancies (TRs) often. Final section concludes the DAWF mechanism performanees through various considered metric anslysis in both time and space.

\section{RELATED WORK}

Encrgy consumption in WSNs is a widely studied issue. and a taxonomy of various categories of WSNs presented in $[6,11]$, and datandriven approaches are related to this work among all the categories.

The prior data collection works have suggested several dataaggregation methods under datadriven approaches, which are categorized as in*network aggregation, compression based and prediction-based data aggregations. TiNA [12] used a clause condition for specifying the differed ranges, if the differed 
range is greater than the specified range between any two values, then the differed result can be reported. otherwise igword. TiNA is more related to our work, as we also used the RV function to find TDRs between every two window stored phenomena among the individual nodes, which is presented in one of our recent research works [10]. CAG [13] proposed a cluster based technique which reports only spatial correlations of eluster nodes by a $\mathrm{CH}$ to the $\mathrm{BS}$, and ignores the individual nodes temporal data. The authors of [14] proposed a another cluster-based method like CAG to build a predictive model on $\mathrm{CH}$ nodes instead of individual sensor nodes and tet the complete computational burden on header nodes itself.

In [15], the authors present a method to build predictive models for exploting the sensed data conclations by a pair of nodes. An auto-regressive (AR) model is presented in [3]. In this regard, nodes can compute a model for the sensed data until a buffer is filled and transmits only the model parameters to the BS. Tan et al from [16]. incestigates the unnecessiry data reduction impact of data fusion on the coverage and detection delay in WSN.

Distributed Soure Coding Using Syndromes (DISCUS) [7] used at framework for distributed data compression by using joint source and channel coding. This method reduces the inter node communication cost for wing a both quantized sourec and correlated side information from every individual node. In [8], the authors proposed a predictive temporal redundant model in data collection, and used it for real-time error correction. And, a source correlated model is suggested in [17] under lossy wireless sensor network with multiple sinks.

Unlke previous works, our proposal awoids the conxider. ation of rich spatio-temporal computationally resource constrained models or predictive models, and designed a simple data redundancy algorithm based on DREAL real datasets. In our considerations, normal nodes sense the envirommental phenomena and filter through DAWF mechanism for $\mathrm{ex}$ ploting TRs and as well as minimizing the redundant data transmissions. Furthermore. DAWF in $\mathrm{CH}$ wodes works with better computational memories, since $\mathrm{CH}$ s are assumed as super nodes for reducing the spatial redundawies (SRs) among the nodes.

\section{Prellminaries}

We consider a cluster bascd sensor network with $n$ normal SNs, which contimously forward the uncorrelated set of data attributes to the $\mathrm{CH}, X(t)=\left(X_{1}, X_{2}, \ldots, X_{m}\right)$ generates the sensed physical phenomena at different time instances $t$, and $s$ number of $\mathrm{CH}$ s as super nodes, which receive the data meserges of SNs, $Y(t)=\left(Y_{1}, Y_{2}, \ldots, Y_{p}\right)$ during SNs window time intervals $r$ and their own sensor reading $X(t)$ at different time instances of $T$. The attribute detection of environmental phenomena $X_{m}$, may be the attributes being sensed by nodes as temperature or humidity or may $b e$ the result of any application phenomena. If the sensor monitoring attributes are either periodic or continuous, it then consider the DAWF mechanism to monitor redundant physical phenomena. This work primarly focuses on the reduction of data redundancies at individual sensor nodes.

\section{A. System Model}

This paper considers that the network molel is a single-hop cluster-based and distributed, as showin in the figure 1. The nodes can compute and process the obtained envirommental data at $t$ time instances through the DAWF mechanism. Since computation is the second highest energy consumer after the communication, even though for computing the algorithm: does not require much energy compared to the communication. The preliminary use case for exploiting temporal redundancies in sensor noles and its experimental study case are presented in [10]. Furthermore, the following implementations hawe been developed for reducing the spatial redundancies and correlations among the nodes.

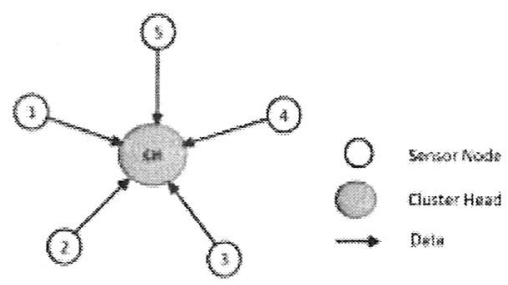

Fig. 1: A cluster-star topology, show the nature of communication between sensor nodes and $\mathrm{CH}$.

In this scenario, $w_{Q}$ keeps the record of nodks received data during the obserwed time interval $R$ and clcared the datia on the basis of FIFO approach after every suecessful data window transmission to the sink node or BS.

Furthermore, we implement the DAWF mechanism in $\mathrm{CHs}$ for finding both SRs and TRs, since $\mathrm{CH}$ s receive the dati messages $Y(t)$ from member wodes as well as their own sensor readings and node physical quantities $X(t)$. We assume that the minimum window $w_{Q}$ size of the $\mathrm{CH}$ is 50 , which can also be variod based on $\mathrm{CH}$ node computational constraints for monitoring nodes data spatially and their own readings for exploiting the TRs. In this case, if sensor nodes have the same applieation monitoring tasks at different periods, then there will be seweral SRs over nodes. Hene, for reducing the SRs among nodes, $\mathrm{CH}$ keeps the data records of all sensor nodes during every round time interval of $R$ at $\mathrm{CH}$ windows. In order to fird SRs, the following expressions Eq. (1) and (2) must be satisfied in $\mathrm{CH}$ nodes.

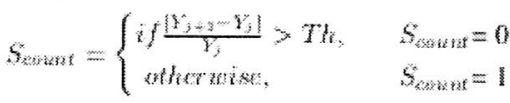

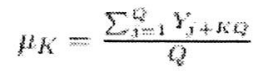

According to the relative variation (RV) of Eq.(1) and Eq. (3), where $\zeta_{\text {coum }}$ represents the spatial count of RV and $T_{\text {cosnt }}$ states the temporal count of RV. If $S_{x x a n}$ is greater than the threshold $T /$ then the $S_{\text {sount }}$ will be equal to 0 otherwise count will be reported as 1 . In this scenario, we set the optimum threshold $T h$ as 0.05 and it can also be 
varied as per the physical phenomena or different application physical activities. And, it can be evaluated by Eq.(2) where $H_{K}$ is the mean value of window at $K$ times which starts from zero. And $j$ is an index of sensor nodes delivered data property at given time instances $t$, and $R$ is the round time interval of $w_{Q}$. And, $Q$ is a window size that can either be fixed or vary based on the nodes computational resources or hash memory. During every $w_{Q}$ of $\mathrm{CH}$ rode, it can compare its member rode value $Y_{3+1}$ with the previous member node value of $Y_{3}$ through the window stored readings by Ea.(1). Moreower, during every window round time interval of $R$ after the data check based on Eq.(1), the previous sensor readings in DAWF can be flushed itself based on First-In-Fist-Out (FIFO) quewe method. In order to exploit the temporal redundancies in both sensor nodes and $\mathrm{CHs}$, and the parameters and variable considerations of TRs have the similar features as like SR expressions. But, the threshold parameters may vary since CHs has two window constraints one window function for nodes received data and another one for $\mathrm{CH}$ nodes sensed physical properties, as like regular sensor nodes. In this scenario, the following constraints should be satisfied in both sensor nodes and $\mathrm{CH}$ for exploiting the temporal redundancies, and the given expressions can be defined as

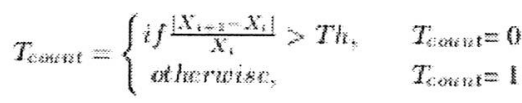

$$
\begin{aligned}
& \mu_{k}=\frac{\sum_{i=1}^{M A} X_{i+k M}}{M}
\end{aligned}
$$

In general applications, most of the obtained environmental datis are either redundant or correlated. We assume that the observed nodes data messages are highly redundant.

\section{B. Adaptive Compression and Predioton Based Medhanism}

The iterative. Algorithm 1 starts by initializing all the variables and their parameters. The core of this algorithm, consist of a data redundancy loop that predicts the node redundant or correlated data with the previous data values. If the current readings differ from the previous readings then only node can forward the data to clusterwead, otherwise the data can bo flushed itself.

The entire algorithm itcratively stored the received data of nodes in an attribute window function, computed and updated in $\mathrm{CH}$ node. In this regard. DAWF mechanism has two time interyals; one as nodes received data time intervals $t$ and another one as window time intervals $R$. Using $\mathrm{RV}$ function (Algorthm 1, line 4) DAWF mecharism examines the window stored data of nodes, whether they are redundant or comelated, if RV is greater than the threshold then it counts and forwards the non correlated data towards the BS. If the window contains the redundant information then (Algorithm, line 8) DAWF uses the mean averaging function to send one appropriate data value rather than all window readings.

On the other hind, DAWF also delivers the non correlated window data as angle packet, the authors of [18] presented that incrasing a payload size (from 1 bytes to 90 bytes) to

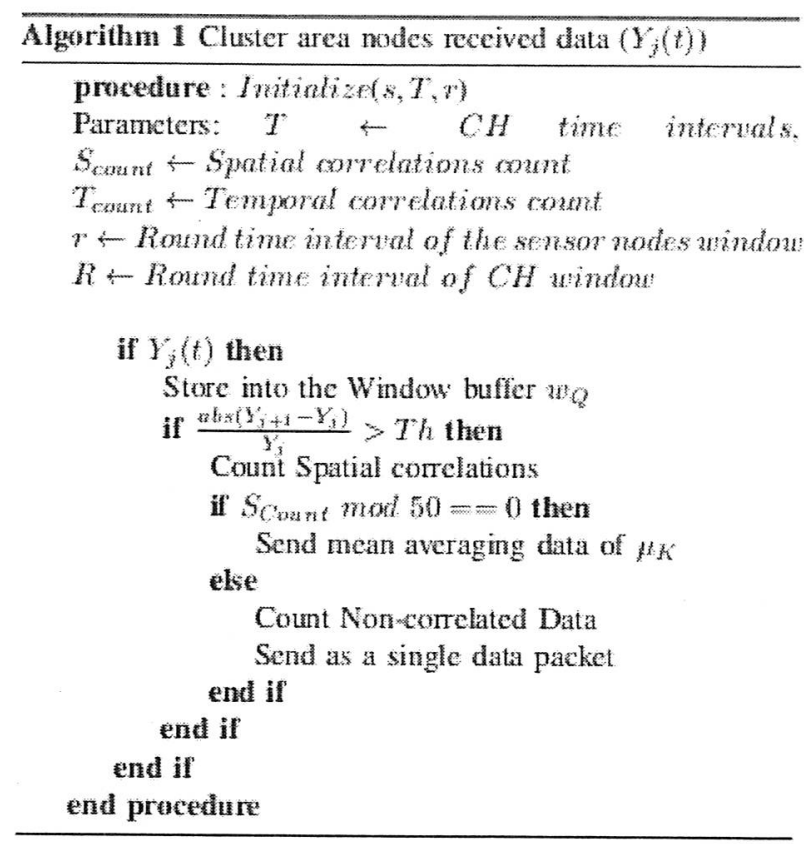

some extent does not add the additional communication costs over the nodes.

\section{EXPERIMENTAL STUd CASE AND RESUlTS}

In order to valuate our models as decribed in the car* lier section, this paper carried out a set of experiments by using real data sets, which were collected by OSAmI-FR WiredWireless Sensor Network (WSN) nodes, contained and supported by DREAL, LCIS research lab from [1].

Simulation results are captured among the test area of sensor nodcs with the help of real sensor network data sets. According to this lab repository, the data values are captured at every one hour, and the readings (ambient temperature, relative humidity, solar temperature, light and $\mathrm{CO}_{2}$ ) are collected from the indoor network deployment of several sensor nodes, between Jamuary 1 and December 30, 2011. Moreover, the sensors appear to be placed randonly in different floors in the housing project. In this experimental study case, we considered two sorts of physical phenomena as solar temperature, and humidity. However, we used in total a year measures of 6 sensor nodes with a considered $\mathrm{CH}$ node.

The primary study case of the simulations are presentol in MATLAB. We have been further developing our proposi. tions in COOJA/Contiki cmulator for measuring the several metric analysis such as message cost and ersergy costs. sine COOJA emulator uses its own developed software's and can be uploaded directly on any COOJA recommended real motes, for instance in our case we consider Tmote-sky for COOJA simulations and TelosB devices for network deployment.

\section{A. Tenporal Peformance Analys to}

This paper presents two sorts of experimental analys is. First one describes the exploitation of data redundancies over the 
collected data of nodes. And, second one focuses on the proposed metric of transmission cost evaluation over both time and space. Since some of the collected data dates and timings differently formatted in the data sets, thus we decided to use 11 months of data for this experimental study to validate the metric of transmission ( $\mathrm{TX}$ ) costs.

In this section, the approach in section 3, and the cluster tree topology that we assume superimposed on the considered amount of sensor nodes. Thus allow us to investigate the outcome of the proposed algorithm in terms of suppressing required number of transmissions as well as cnergy saving.

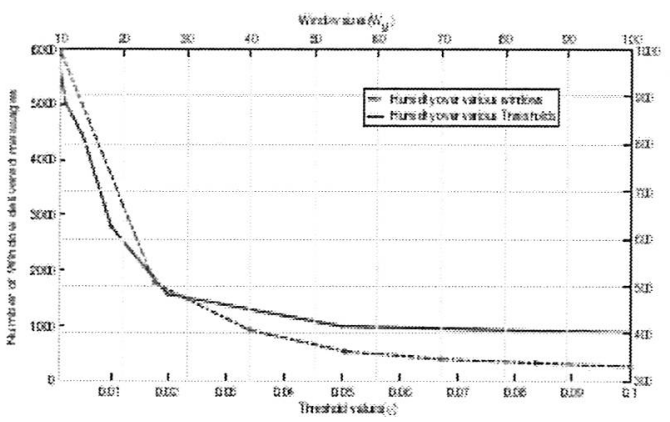

Fig. 2: DAWF different parameter performances with various thresholds $T h s$ over different $w_{M}$ sizes of temporal dati

Figure 2 illustrates the spationtemporal DAWF mechanism of considered physical quantities by computing through var ious thresholds $\eta$ of 0.001 to 0.1 with a fixed window size of 10 , and at a different window sizes of 10 to 100 with an optimum threshold of 0.05, which was demonstrated in below subsections. In this scenario, DAWF presents a comparatiwe analysis between nodes threshold parameters and their window constraints to validate that increasing a window size according to the nodes computational constraints would be always ben eficial. Figure 2 clearly shows that node window constraints indeed plays a significant role to reduce the data bandwidth without losing any significant data. However, this is the case for temporal exploitation in nodes. But, in spatial correlations exploiting of the nodes in $\mathrm{CH}$, threshold parameters are playing the major role for delivering finest data performanees compared to the window sizes.

Figure 3 exploits the various window performances together with different throxhold variations in time. In this scenario, we show how the window performanes gradually increases while turing the $\eta$ and $w_{M}$ parameters combinedly compared to the individual variations as shown in the figure 3 . Which then proved that window performances of the temporal nodes are directly proportional to the optimum thresholds of the sensor nodes or their dynamicity based on the different phenomena.

Figure 4 and 5 shows the transmission (TX) cost metric performances over the considered network area. The reliability of the proposed aggregative models has been assessed by computing the transmission $(T X)$ cost. The proposed metric of $T X_{\text {cost }}$ is given by Eu. (5) to calculate the total amount of (perw centage) of saved transmis sions per node while exploiting the temporal data redundancies. As shown in figure 4, and figure 5 .

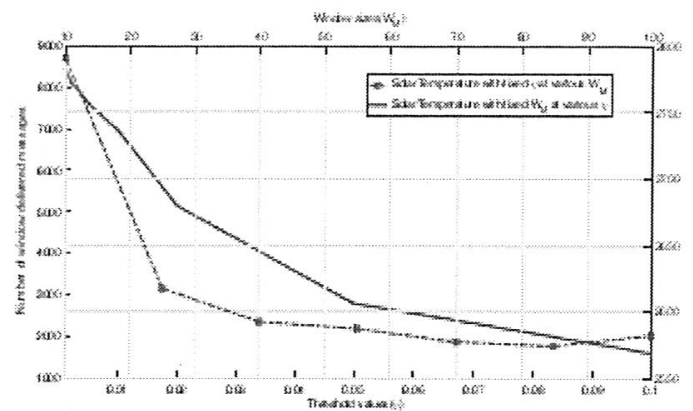

Fig. 3: CH DAWF performances of solar temperature over various window sizes

which also explored the different phenomena performances of nodes throught the $T X_{\text {wast }}$ metric. In figure 4 , node can reduce a minimum of $88.62 \% T X_{\text {sos }}$ in humidity by computing through the DAWF algorithm, and the maximum of $99.62 \%$ with the consideration of higher window interval as 100 and with an optimum threshold parameter of 0.05 . According to the various threshold parameters, where DAWF reduced a minimum of $39.76 \%$ redundant transmissions by a minimum wirdow size and threshold of $10,0.001$, respectively. Thus, the maximum of $8989 \%$ redundant transmissions was suppressed by a maximum threshold parameter of 0.1 at a constant optimum window size of 10 . However, when it comes to solar temporal phenomena in figure 5, the DAWF algorithm of various window sizes shown less impact, as a minimum window size of 10 reduced $63.70 \%$ and the window size of 100 reduced $66.19 \%$ only by a constant threshold of 0.05 . On the other hand, threshold parameters of solar temperatures showed better results compared to the various window sizes. With the minimum constant window size of 10 by a minimum threshold of 0.001 , reduced $3.22 \%$, and a maximum threshold parameter of 0.1 suppressed 79.94 of $T X_{\text {rasi }}$. In this scenario, we noticed that the performanes of various window sizes shown more or less like same results compared to the threshold parameters, because the nodes have less correlated data of solar temperature, since the monitored data were obtained by outdoor sensors. Therefore, the following metric is being

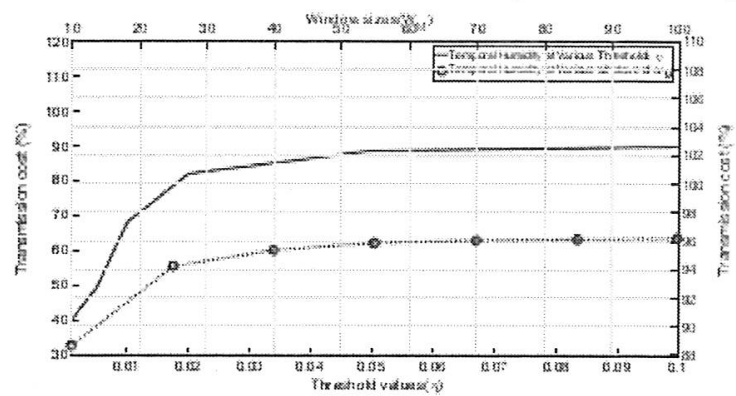

Fig. 4: $\mathrm{CH}$ DAWF temporal data relative humidity $\mathrm{TX}$ cost metric performances over various thresholds and the various window sizes. 


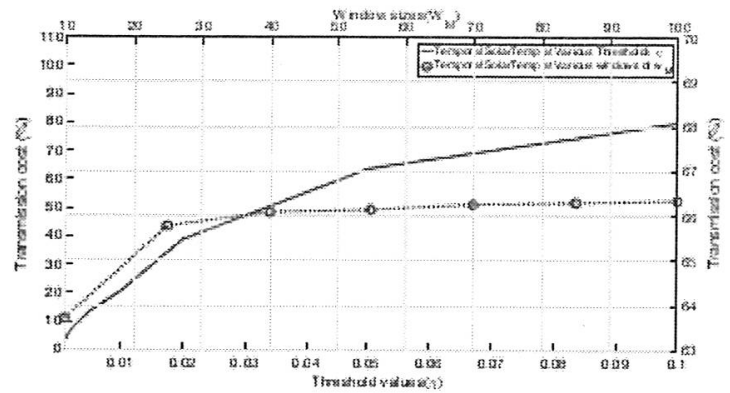

Fig. 5: Nodes TX cost metric performances Solar Temperature.

used, in order to present the nodes $T X_{f w s}$ performanes after suppressing the redundant data transmissions by the window mechanism, and the $T X_{\text {was }}$ metric can be derived as:

$$
T X_{\text {rast }}=\left(1-\frac{\text { Total window sent messages }}{\text { Total whindon read messages }}\right) \times 100
$$

\section{B. Spatial Perfomance Analys is}

In order to suppress the redundant data and the required amount of transmissions spatially, we use the $\mathrm{CH}$ node to find the correlations among the considered sensor nodes sent data. Since we used different physical sensor quantities in nodes, in order to find and evaluate their spatial data by using pre-filtration techniques in $\mathrm{CH}$ node as like temporal analysis, which were also collected by both indoor ard outdoor deployments.

Figure 6 exploits the nodes spatial phenomena over various window sizes and at different threshold parameters. Figure 6 is shown that the threshold parameters only delivered a varied spatial performanees among different spatial phenomena of the nodes. However, in figures 6 and figure 7 window size

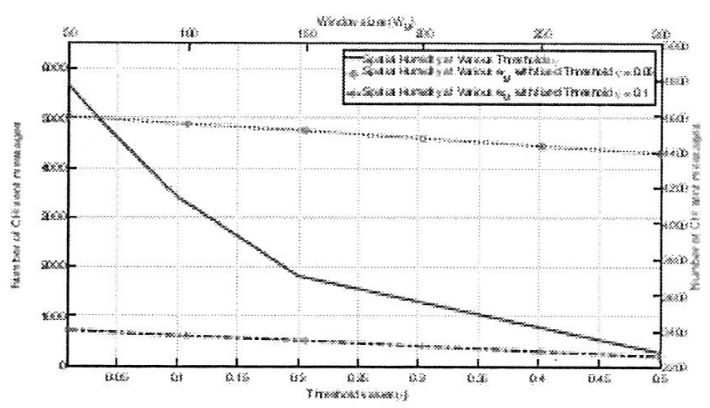

Fig. 6: CH DAWF spatial relative humidity performances over various threshols through the window sizes.

constraints did not show any greater impacts spatially while compared to the threshold parameters in $\mathrm{CH}$, like nodes, as they performed well in exploiting temporal redundancies. In this case, whatever the window size is maximized then the delivered data are showing slight variations in window performances, but many remain constant, as shown in $6(b)$. Because, $\mathrm{CH}$ collected data from the nodes are not either highly redundant wor correlated. In this scenario, we presented the window varying performanees with two fixed optimum thresholds of 0.05 and 0.1 . Figure $8(a)$ and $8(b)$ exploits the spatial $T X_{\text {ressts }}$ of different nodes received phenomena in $\mathrm{CH}$. In this metric analysis is given by Eq.(5), we carricd out the performances as threshold over window size variations. In Figure $8(\mathrm{a})$ and $8(\mathrm{~b})$, where $\mathrm{CH}$ suppressed the amount of $5.19 \%$, and $2.90 \%$, respectively by computing through a minimum threshold of 0.01 , and the maximum threshold of 0.5 shown the performances as $95.25 \%$, and $78.65 \%$, respec tively. On the other hand. DAWF window sizes delivered the performanes, as a minimum amount of reduced transmis sions are $22.95 \%$ and $10.75 \%$ by an optimum threshold of 0.03 and with the maximum window constraints of 300 delivered the performances as $26.34 \%$ and $13.15 \%$ among the nodes. Which is clearly demonstrated that the threshold parameters always have a high impact compared to the window sizes, when it comes to spatial phenomena for delivering outperforming results. For instance, according to the data sets $x_{\times}$nodes

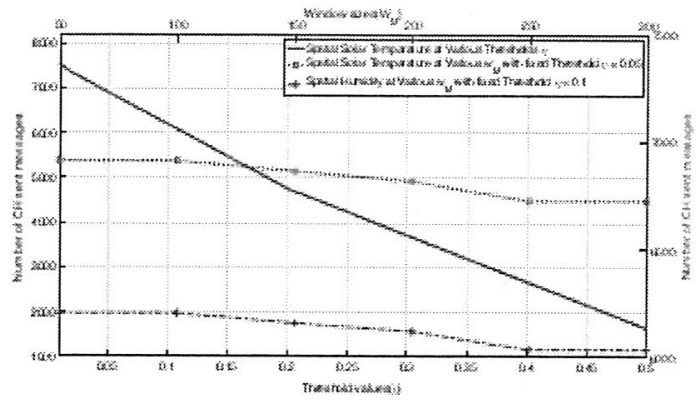

Fig. 7: CH DAWF spatial performances of solar over various thresholds through the window sizes.

follow the periodic transmission system as every bour, then it transmits total considered nodes measures of their sensed phenomena at given schedukd intervals. In the contrast, using DAWF mechanism at a minimum window size of 50 through $\mathrm{CH}$ where it reduces $22.95 \%$ and $10.75 \%$ amount of redundant messages out of total different received data nodes phenomena by computing through the constant threshold parameter of 0.05. Which is clearly proven by Eq. (5) that CH DAWF is necessary to hawe in head nodes, and it can still suppress the data correlations among the nodes spatial data.

Furthermore, temporal redundancies in $\mathrm{CH}$ node at various thresholds with different window sizes of $w_{M}$ also performed same as sensor nodes, which presented and explained in the section 4.1. Which is also clearly stated that temporal redundancies clearly depends upon the window sizes rather than threshold values. Where it suppresses upto $90 \%$ temporal redundances in $\mathrm{CH}$ received data of nodes by compung through the optimal thresholds and maximum window sizes of the CHs. Which is obvious, if the window sizes are bigger then the stored data and comparison between every two window stored readings can be drastically increased. In order to show the perfomance analysis of the proposed approach in terms of energy saving, as briefly illustrated in the above results. The DAWF can efficiently suppress required number of transmissions with respect to the periodic or delay-tolerant 


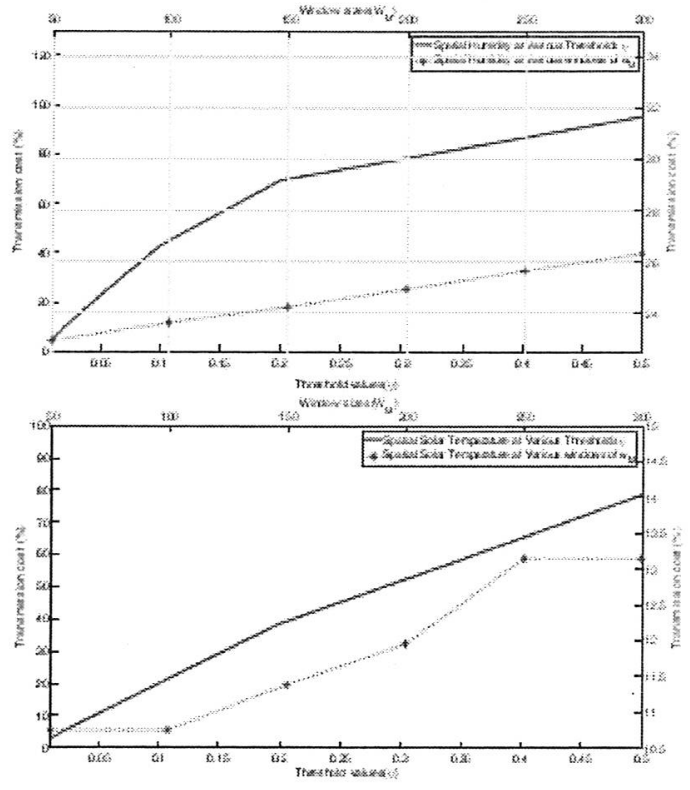

Fig. 8: (a) CH DAWF TX cost metric performances of humid ity over various thresholds through the window sizes (b) $\mathrm{CH}$ DAWF TX cost metric perfomanes of solar temperature over various thresholds through the window sizes.

approach where each node forwards its obtained data whenever it detects to the collecting node or BS.

\section{CONCLUSION AND FUTURE WORKS}

This paper presented a fully distributed spatio-temporal nowel approak in sensor rodes, which aimed to keep the Wireless Sensor Network as energy efficient as possible. The use of presiltration data techniques for exploiting the physical quantities allowed to reduce the communication costs ower nodes, and thus maximizes the sensor nodes lifetine, by adaptively tuning their data rate. The use of simple pre- filtration techniques rather than complex computationally cxpensive models was the basic idea to design a window technique, which adapts the dynamicity regardless of any particularly monitored data. The experiments carried out on real data sets shown better performances, in particular when the threshold set to $5 \%$, the overall amount of energy saving in $\mathrm{CH}$ node among the received nodes data phenomena are $22.9 \%$ and $10.75 \%$, respectively. And thus, reducing the temporal redundancies in nodes, it saves upto $90 \%$ of redundant data transmissions. Furthemore, our approach requires to make a comparison between a much lower sampling rate than what chosen for the original configuration, in terms of measuring rellability.

The ongoing work still need to make further improvements, in order to make the approach to have complete adaptivity. by which a node can tune the threshold parameters or window constraints based on monitored data attributes. In particular, the algorithm refinement is required. especially when the data patterns are irregularly ineremented, but correlation between near by the data values. In this case, therefore non-correlated data counter should be effective in DAWF to list the all non correlated data as single packet. Further experiments are also being examined, in order to present the comparative analysis with different metric considerations to measure the system performances, especially in terms of quality of data, latency, message costs in $\mathrm{CH}$ nodes if the window constraints are high, and energy cost in overall network.

\section{REFERENCES}

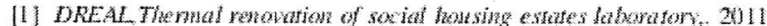

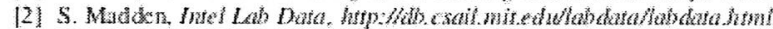
3 ?า4.

[3] D. Tulme and S. Madken, PAQ. Time series forecasting for apprexi-

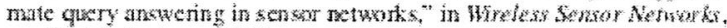
pp. $21-37$. Springer, 2006

[4] E-o. Blass, J. Honxber, and M. Zitterbart, "Analyzing Data Prediction in Wireless Sensar Networks" in IEEE W Ricular Fermology Coryfer.

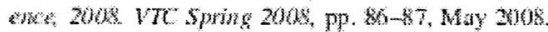

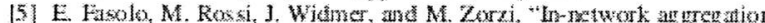
techniques for wireless sensor networles: a surwey" Hireless Conmunicaiwa, JEEE, wol. 14, no. 2 pp. $70-87,2007$.

[0] G. Anastasi. M. Conti, M. Di Franceso, and A. Paxsarella. Escry eonservation in wireless sensor retuerks: A suryey," Ad how mowh. wol. 7, w. 3, pp. $537-568,200 \%$

(7) \$. Prathan ark K. Ramblandran, "Distributed source codinge using

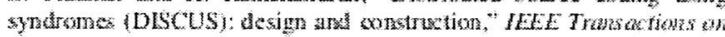
Whomation Theor, wi. 49, no, 3, pp. 626-643, 2003.

18 I S. Makwopathyay, C. Schurners, D. Parizrahi, and S. Dey, "Model Based Teohniques for Data Reliability in Wireless Semora Netwarks." IEEE Transactions wh Mobife Coupuring, vol. \& pp. $528-543$ Apr 2)

(9) L. Warg amd A. Deshparke, "Predictive madeling based dina collestion

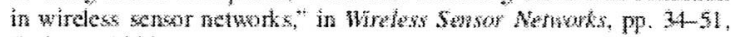
Springer, $200 \mathrm{~s}$

no] S. Kandukari, J. M. Lebreton, N. Murak?, R. Larion, ande 1. D. LanSun-Luti, "Exwryy-Efficient Chater-Based Protscal using An Adaptiye Dafa Agregative Wirkow Function (A-DAWF) for Wireless Senwor

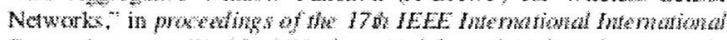

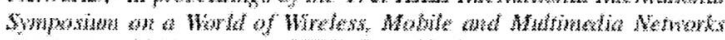
(Wo WMa 2016), pp. 1-4, LEEE, Jume 3016 .

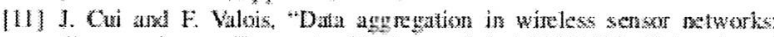
Compressing or Forecasting" Research hal-0)061598. University of Lyon, Irria, WNSA Lyon, CrI, France, Sept. 2013

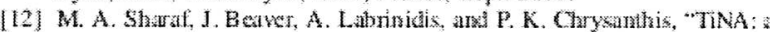

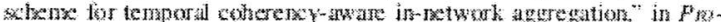
ceeding of the 3 il ACM internariond workhop on Data engineering

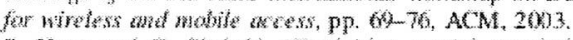

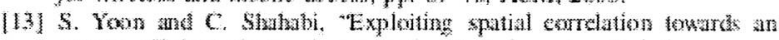
exergy efficien clessiered aggregation tedsvigue (eag)|wireless senson

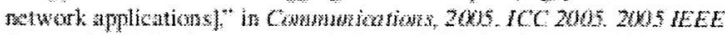
Hiterational Conference on wal. 5, pp. 3307-3313. IEEE, 2015.

114) A De Panla, G. Lo Re, F. Milazwo, and M. Orolari, "Predictive models tor energy saving in wireles sernor netwals." in Whid of Wirdesw.

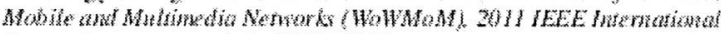
Symposiam ow $a$, pp. 1-6. EEE, 311 .

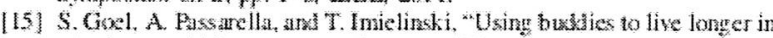
a boring wotd |sensor network protocoll," in Penvasive Compungang and

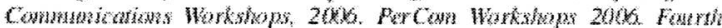

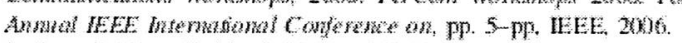

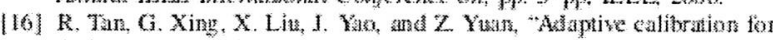
fusion-based wireless sencor networks." in INFOCOM, 2010 Proced Migg IEEE, pp. 1-9, IEEE, 2010

(17) M. Rowhanei and M. Valiporr, "Ereny-Efliejern Dana Gotbering ower Wirelese Sensor Networks: Correlated Swares aw Lossy Chamels," pp. 44) 451, IEEE, May 2009 .

(18) $C$. Hata and J. Wilke, "Erergy evaluations in wircless sensor network:

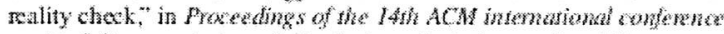

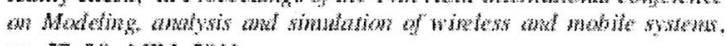
mp. $27-30, \mathrm{ACM}, 2011$. 